\title{
Value of sentinel lymph node mapping using a blue dye-only method in gastric cancer: a single-center experience from North-East Hungary
}

\author{
Dezső Tóth $\cdot$ Zsolt Kincses · János Plósz • \\ Miklós Török · Ilona Kovács · Csongor Kiss • \\ László Damjanovich
}

Received: 20 January 2011/ Accepted: 29 March 2011/Published online: 3 May 2011

(C) The International Gastric Cancer Association and The Japanese Gastric Cancer Association 2011

\begin{abstract}
Background Forty percent of patients with gastric cancer have unnecessarily extended lymph node dissections with higher rates of morbidity and mortality than those in nonextended procedures. Successful sentinel lymph node (SLN) mapping may help to reduce the number of extended lymphadenectomies.

Methods SLN mapping was investigated by a blue dyeonly method in patients with gastric cancer. The first cohort of patients $(n=16)$ were marked submucosally by an endoscopist and in the second cohort of patients $(n=23)$ a subserosal injection was performed by the surgeon.

Results Thirty-nine patients, all Caucasians, underwent gastric resection or total gastrectomy with SLN biopsy using patent blue-dye mapping and modified D2
\end{abstract}

D. Tóth $(\bowtie) \cdot$ Z. Kincses

Department of General Surgery, Kenézy Teaching Hospital,

2-26 Bartók Street, Debrecen 4043, Hungary

e-mail: detoth@gmail.com

J. Plósz

Department of Internal Medicine, Kenézy Teaching Hospital,

2-26 Bartók Street, Debrecen 4043, Hungary

M. Török · I. Kovács

Department of Pathology, Kenézy Teaching Hospital,

2-26 Bartók Street, Debrecen 4043, Hungary

C. Kiss

Department of Pediatric Haematology-Oncology, Institute of Pediatrics, Medical and Health Science Center, University of Debrecen, 98 Nagyerdei Road,

Debrecen 4012, Hungary

L. Damjanovich

Institute of Surgery, Medical and Health Science Center,

University of Debrecen, 98 Nagyerdei Road,

Debrecen 4012, Hungary lymphadenectomy. The mapping procedure and the lymphadenectomy were supervised by the same surgeon. A total of 770 lymph nodes were removed and examined. The mean number of blue nodes was 4.3 per patient. In 22/23 cases at least one SLN showed tumor involvement. The sensitivity of SLN mapping was $95.7 \%$, the false-negative rate was $4.3 \%$, and the specificity was $100 \%$. The negative predictive value was $93.8 \%$ and the positive predictive value was $100 \%$. In cases of $\mathrm{T} 1$ and $\mathrm{T} 2$ tumors the sensitivity was $100 \%$. We found the two marking methods (submucosal vs. subserosal) to be equivalent and there was no side-effect of the blue-dye mapping.

Conclusions Our results suggest that SLN mapping with blue dye alone represents a safe procedure that seems to be adaptable for non-obese patients undergoing open surgery for gastric cancer in the Eastern European region. The procedure has high sensitivity and specificity, especially in cases of $\mathrm{T} 1$ and $\mathrm{T} 2$ tumors.

Keywords Gastric cancer - Patent blue V .

Sensitivity and specificity $\cdot$ Sentinel lymph node biopsy

\section{Introduction}

The presence of lymph node metastases is the most important predictive factor for survival in gastric cancer following curative resection [1, 2]. A large portion of Western European patients undergoing R0 resection do not have nodal involvement [3]. More than one-third of patients with gastric adenocarcinoma have unnecessarily extended lymph node dissections with higher rates of morbidity and mortality than those in non-extended procedures. Several studies have shown increased perioperative morbidity and mortality with D2 versus D1 lymph 
node dissection [4-6]. Successful sentinel lymph node (SLN) mapping may help to define those patients who would and those who would not benefit from an extended lymph node dissection in association with gastrectomy.

SLN mapping has become a standard procedure in breast cancer and malignant melanoma [7, 8]. The injection method and selection of tracers for SLN mapping in gastric cancer remain controversial. Studies from Japan and from Western Europe have demonstrated a high detection rate $(>95 \%)$ and a sensitivity of $90-95 \%$ for SLN biopsy in patients with gastric cancer [9-13]. Keeping the well-known differences between Asian and European gastric cancer phenotypes in mind [14-16], we have evaluated, for the first time, the validity of SLN mapping using a blue dye-only method, in a standardized prospective study of patients with gastric cancer in Hungary.

\section{Patients, materials, and methods}

The study was conducted prospectively from February 2008 to December 2010 at the Department of Surgery of the "Gyula Kenézy" Teaching County Hospital, Debrecen, Hungary. Thirty-nine consecutive patients underwent open gastric resection with blue-dye mapping and modified D2 lymph node dissection. Exclusion criteria were distant metastases and involvement of the surrounding organs (T4). After laparotomy, the lesser sac was opened (if this was needed) and $4 \times 0.5 \mathrm{~mL}$ blue dye (Bleu Patenté V 2.5\%; Sodique Guerbet, F-95943 Roissy CdG, Cedex, France) was injected in four quadrants around the tumor. The first cohort of patients $(n=16)$ were marked submucosally by an endoscopist, and in the second cohort of patients $(n=23)$ the injection was performed by the surgeon subserosally. Lymphatic drainage was observed for up to $10 \mathrm{~min}$ following the injection. Blue-stained lymph nodes were marked, and a modified D2 lymph node dissection was performed after gastric resection or total gastrectomy. The staining method and the lymphadenectomy were supervised by the same surgeon. The position of lymph nodes was marked according to the Japanese classification of gastric carcinoma [17]. SLNs were examined in frozen sections intraoperatively. Postoperatively, the lymph nodes were sectioned at 2- to 3-mm intervals and hematoxylin and eosin (HE) staining was performed; in special cases (signet-ring cell carcinoma), immunohistochemistry examinations were performed, with DAKO Monoclonal Mouse Anti-Human Cytokeratin (clone AE1/ AE3; dilution 1:30; Dakocytomation; Glostrup; Denmark). Only negative sentinel nodes were examined for micrometastasis. The median follow up was 18 months (range 1-35 months).
The detection rate was calculated as the number of successful identifications of SLNs in relation to the total number of SLN biopsy procedures.

The sensitivity was given as the number of patients with positive SLN among the total number of patients with histopathological evidence of lymphogenous tumor spread. The false-negative rate was defined as $100 \%$ minus sensitivity. Specificity was calculated as the number of negative SLN biopsy results in relation to the sum of negative SLN biopsy results plus false-positive results. The negative predictive value was calculated as the number of negative results per the sum of negative results and false-negative results, while the positive predictive value was quantified as the number of positive results per the sum of positive results plus false-positive results. Statistical analysis of equivalence between the two marking methods was based on calculating the ratios of test performance indicators (those of the subserosal method divided by those of the submucosal procedure). Equivalence was established when the $90 \%$ exact confidence interval (CI) around such a ratio was fully contained within the range 0.8-1.25.

\section{Results}

We investigated 39 consecutive patients, all Caucasians, 20 females and 19 males with a mean age of 64.3 (range 50-80) years. The average body mass index (BMI) was 22.8 (range 17.1-27.6). Fourteen patients had signet-ring cell carcinoma, 19 patients had moderately differentiated adenocarcinoma, and 6 patients had poorly differentiated adenocarcinoma. The tumor was localized in the upper third of the stomach (U) in 8 cases, in the middle third of the stomach (M) in 9 cases, and in the lower third of the stomach (L) in 22 cases. The depth of invasion was T1 in 9, $\mathrm{T} 2$ in 11, and T3 in 19 patients. Total gastrectomy was performed in 14 patients, and subtotal gastrectomy was performed in 25. A total of 770 lymph nodes were removed, and 19.7 lymph nodes per patient were examined on average (range 10-38 lymph nodes per patient). The mean number of blue nodes was 4.3 per patient (Table 1).

The frequency of lymph-node marking was observed in relation to the tumor position (Figs. 1, 2, 3). In 38 cases SLNs were successfully identified, resulting in a detection rate of $97.4 \%$. Sixteen patients $(41 \%)$ were histologically node-negative; in 15 patients both SLNs and non-SLNs were negative. In one case there was no sign of any marking; the BMI of this patient was 26.8 .

In $22 / 23$ cases at least one SLN showed tumor involvement, with a false-negative rate of $1 / 23(4.3 \%)$. The sensitivity was $95.7 \%$, and the specificity was $100 \%$. The negative predictive value was $93.8 \%$ and the positive predictive value was $100 \%$ (Table 2). 
Table 1 Characterization of patients with gastric cancer and sampling procedure

\begin{tabular}{ll}
\hline Number of patients & 39 \\
Tumor sites & \\
Upper & 8 \\
Middle & 9 \\
Lower & 22 \\
Depth of invasion & 9 \\
T1 & 11 \\
T2 & 19 \\
T3 & \\
Nodal involvement & 16 \\
N0 & 13 \\
N1 & 8 \\
N2 & 2 \\
N3 & 770 \\
Total number of dissected lymph nodes & 19.7 (10-38) \\
Median number of dissected lymph nodes (range) & 168 \\
Total number of SLNs & 4.3 (1-15) \\
Mean number of SLNs (range) &
\end{tabular}

Based on International Union Against Cancer (UICC) TNM classification of malignant tumours. [28, 6th edition]

SLN sentinel lymph node

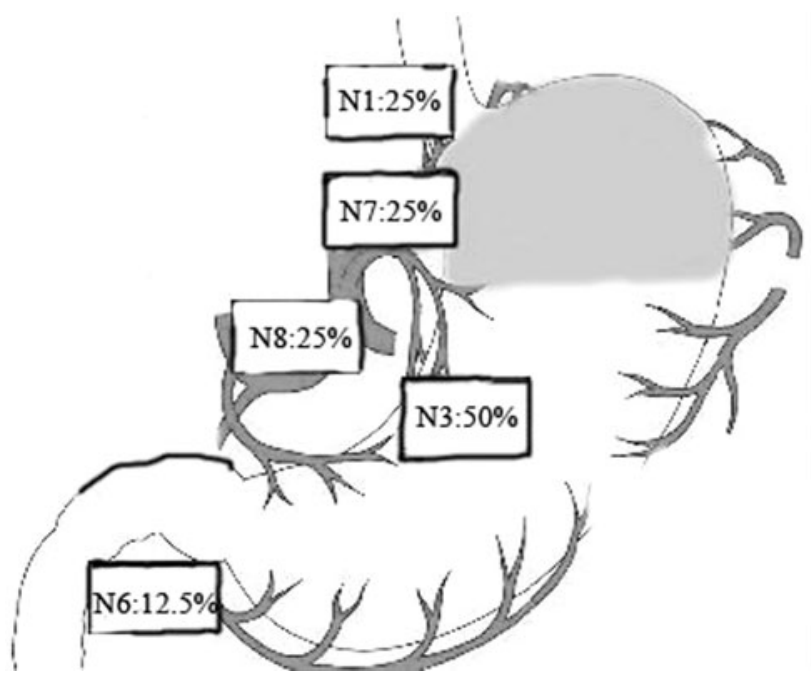

Fig. 1 Frequency of lymph node marking in cases of upper-third tumors

The SLNs were in the D1 compartment in 22 cases (57\%), in both the D1 and D2 compartments in 12 cases (32\%), and in the D2 compartment only in four cases $(11 \%)$.

Regarding lymph node involvement in the patients, exclusively SLNs were positive in $6 / 23$ cases $(26 \%)$; in two of these six cases these nodes were found only in the D2 compartment. In 17 cases other lymph nodes were also involved.The D1 compartment only was involved in 8/17

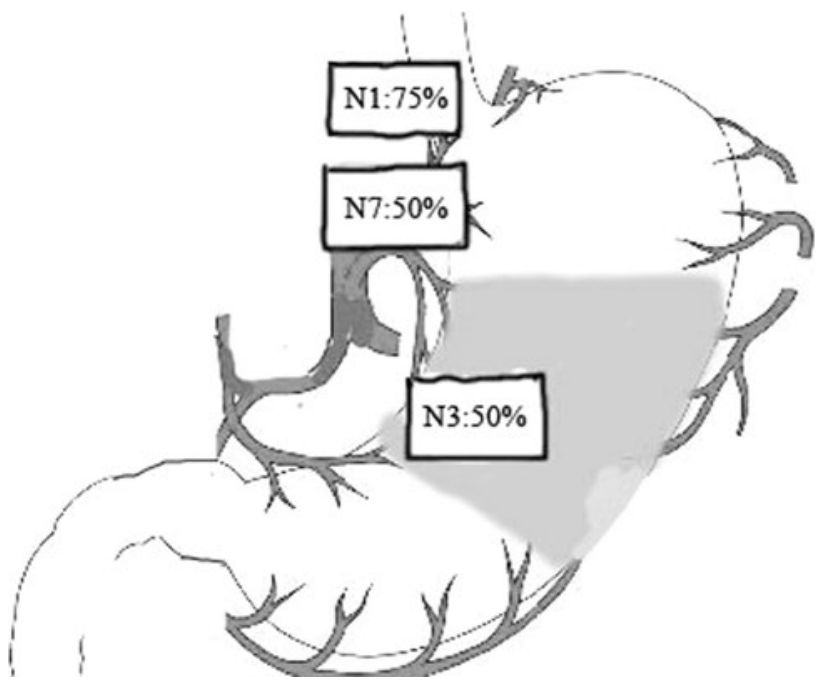

Fig. 2 Frequency of lymph node marking in cases of middle-third tumors

(47\%) cases, and the D1 and D2 compartments were involved in $9 / 17(53 \%)$ cases. In the T1 tumor group $(n=9)$, SLNs were not involved. In the T2 group $(n=11)$, 8 patients were SLN-positive and 4 of them were nonsentinel-positives, as well. In the T3 group, only 5/19 $(26.3 \%)$ patients were lymph node-negative. In the single false-negative case, the tumor was a T3 in extension; grade 3 with perineural invasion was found histopathologically and macroscopically involved lymph nodes were found during the operation. In the fifteen SLN-negative cases micrometastasis was not found in the sentinel nodes. Results of the intraoperative SLN frozen sections correlated completely with the postoperative pathological findings.

The submucosal and subserosal marking methods were proven to be equivalent in detection rate, sensitivity, and specificity based on the $90 \% \mathrm{CI}$ of the ratio of indicators (Table 3). There was no side-effect of the blue-dye mapping. The postoperative period was uneventful in all 39 patients, without any surgical or non-surgical complication.

During the follow-up period of 18 months we lost 6 patients due to progressive disease. Four of the patients who succumbed had pT3 tumors with lymphovascular invasion and two patients had pT2 signet-ring cell carcinoma. All six patients had positive SLNs; in four cases only one sentinel node was positive.

\section{Discussion}

Dutch and British prospective randomized trials found higher morbidity and mortality rates following extended lymph node dissection in patients with gastric cancer when compared to the rates in those who underwent D1 dissection only [4, 5]. Forty percent of Western European 
Fig. 3 Frequency of lymph node marking in cases of lowerthird tumors

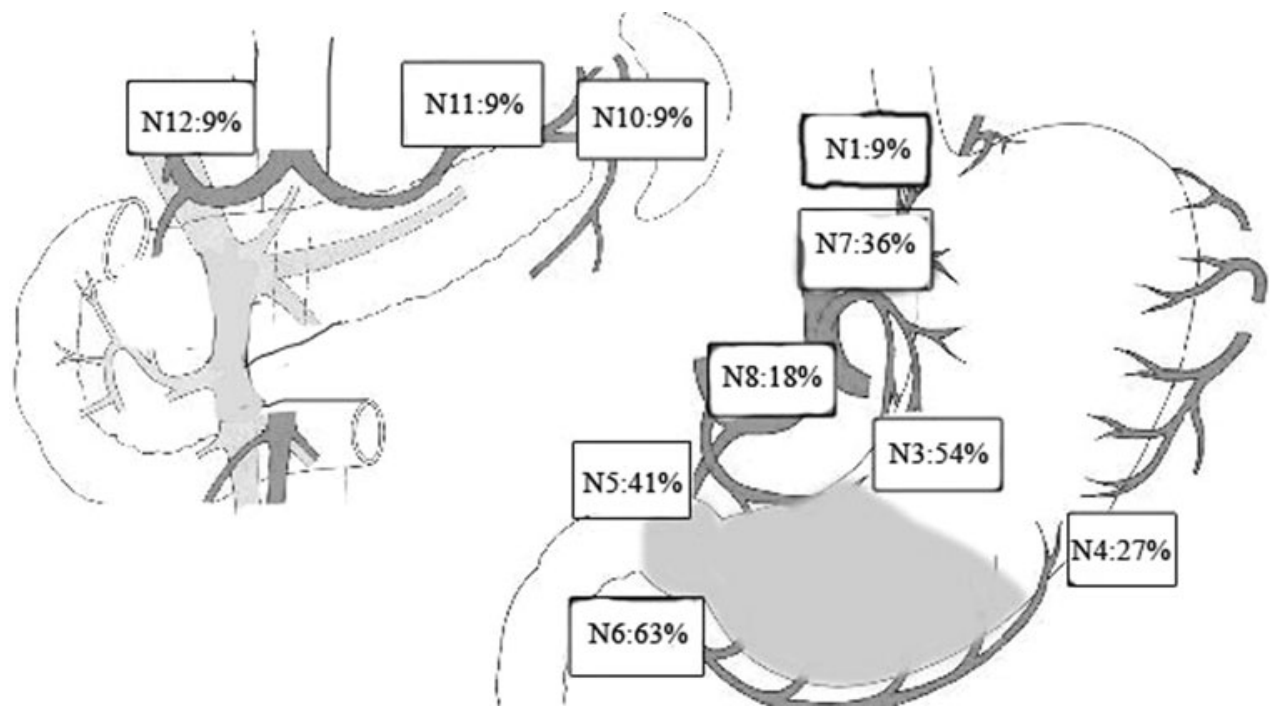

Table 2 Sensitivity and specificity of SLN mapping procedure using blue dye-only method in patients with gastric cancer

\begin{tabular}{ll}
\hline Detection rate $(\%)$ & $38 / 39(97.4 \%)$ \\
False-negative rate $(\%)$ & $1 / 23(4.3 \%)$ \\
Sensitivity $(\%)$ & $22 / 23(95.7 \%)$ \\
Specificity $(\%)$ & $15 / 15(100 \%)$ \\
Negative predictive value $(\%)$ & $15 / 16(93.8 \%)$ \\
Positive predictive value $(\%)$ & $22 / 22(100 \%)$ \\
\hline
\end{tabular}

Table 3 Sensitivity and specificity of SLN mapping procedure in relation to the marking method (submucosal versus subserosal)

\begin{tabular}{llll}
\hline & $\begin{array}{l}\text { Submucosal } \\
(n=16)\end{array}$ & $\begin{array}{l}\text { Subserosal } \\
(n=23)\end{array}$ & $\begin{array}{l}\text { Result } \\
(90 \% \mathrm{CI})\end{array}$ \\
\hline Mean number of SLNs & 4.1 & 4.9 & \\
Detection rate (\%) & 100 & 96 & Equivalent \\
False-negative rate (\%) & 0 & 7.7 & \\
Sensitivity (\%) & 100 & 92.3 & Equivalent \\
Specificity (\%) & 100 & 100 & Equivalent \\
\hline
\end{tabular}

Criterion of equivalence: $90 \%$ confidence interval (CI) should be fully within the range $0.8-1.25$ (detection rate $0.889-1.029$, sensitivity $0.809-1.053$, specificity $1.000-1.000)$

patients with R0 resection have an unnecessary extended lymph node dissection [3]. The preoperative diagnostic tools that define the patient subpopulations which would and would not benefit from an extended lymph node dissection have low sensitivity and specificity. For detecting pathological lymph node involvement the sensitivity, specificity, and accuracy of spiral computed tomography is $73.1,50.0$, and $84.2 \%$, respectively [18], and endoscopic ultrasonography has an accuracy of $68.6 \%$, with a sensitivity and a specificity of 66.7 and $73.7 \%$, respectively [19]. Recent studies have shown that SLN mapping has a high detection rate (>95\%) and a sensitivity of $90-95 \%$ [9-13]; therefore, SLN mapping may help in avoiding unnecessarily radical dissections.

Various mapping techniques have been described in the literature. The injection method and selection of tracers for SLN mapping remain controversial. Some authors use dye alone [12, 20,21] and others apply 99m Tc tin colloid imaging [9], while others use these two techniques in a parallel fashion [22]. Yaguchi et al. [23] and Lee et al. [24] have compared the subserosal with the submucosal labeling method without finding any significant differences. We have had an excellent outcome of SLN mapping with the blue dye-only method in breast cancer. Our detection rate was $92.6 \%$ and the falsenegative rate was $2.6 \%$ [25]. We tried to adopt this method for SLN marking in gastric cancer because conditions for radioguided mapping at our hospital are less than optimal. This study represents the first experiences using the blue dye-only (submucosal versus subserosal) SLN method for patients with gastric cancer from the Central-Eastern European region. Although it is difficult to draw definitive conclusions due to the small size of the series in our prospectively controlled study; we detected SLNs in $97.4 \%$ of patients with gastric cancer with $95.7 \%$ sensitivity and $100 \%$ specificity using the blue dye-alone technique. The false-negative rate was $4.3 \%$. Although, in our series, this meant only one patient (stage T3, perineural and lymphovascular invasion), this indicates that even if labeling is negative, a more extended lymph node removal is necessary in advanced cases. The two cases of positive SLNs in the D2 compartment only call attention to the possibility of skip metastases. Using the blue dye-alone marking method we can avoid an inadequate surgical procedure by applying a limited lymph node dissection. Sensitivity was $100 \%$ in our $\mathrm{T} 1$ and $\mathrm{T} 2$ groups.

In our investigation, only the negative sentinel nodes were examined for micrometastasis, because of the widely 
recognized concept that lymphatic micrometastasis spreads first to sentinel nodes in gastric cancer [26]. Equivalence between the submucosal marking and the subserosal injection methods was proven in this study. Sometimes the identification of SLNs in obese patients is difficult with subserosal injection due to the feathering of blue dye in the fatty tissues. It is well known that the BMI affects SLN detection [27], as was found in our investigation; the BMI of the single non-marked patient was higher than average (26.8 vs. 22.8). In patients with non-palpable tumors we preferred the endoscopic injection. The blue-dye marking method is suitable mainly for open procedures, because real-time observation and mobilization of the stomach can readily be achieved.

In conclusion, SLN mapping with blue dye only seemed to be a safe and feasible procedure in our hands for nonobese patients during an open surgical procedure; such procedures can reliably be performed in other Eastern European surgical departments not appropriately equipped for radio tracer detection. Validation of this single-agent method and the subserosal injection of the tracer requires further evaluation.

\section{References}

1. Siewert JR, Bottcher K, Stein HJ, Roder JD, et al. Relevant prognostic factors in gastric cancer: ten-year results of the German Gastric Cancer Study. Ann Surg. 1998;228:449-61.

2. Kim JP, Kim YW, Yang HK, Noh DY, et al. Significant prognostic factors by multivariate analysis of 3926 gastric cancer patients. World J Surg. 1994;18:872-7.

3. Kooby DA, Suriawinata A, Klimstra DS, Brennan MF, Karpeh MS. Biologic predictors of survival in node-negative gastric cancer. Ann Surg. 2003;237(6):828-37.

4. Bonenkamp JJ, Songun I, Hermans J, Sasako M, Welvaart K, Plukker JT, et al. Randomised comparison of morbidity after D1 and D2 dissection for gastric cancer in 996 Dutch patients. Lancet. 1995;345:745-8.

5. Cuschieri A, Weeden S, Fielding J, Bancewicz J, Craven J, Joypaul $\mathrm{V}$, et al. Patient survival after D1 and D2 resections for gastric cancer: long-term results of the MRC randomized surgical trial. Surgical Co-operative Group. Br J Cancer. 1999;79:1522-30.

6. Dent DM, Madden MV. Price SK Randomized comparison of R1 and R2 gastrectomy for gastric carcinoma. Br J Surg. 1988;75: $110-2$.

7. Nieweg OE, Bartelink $\mathrm{H}$, et al. Implications of lymphatic mapping for staging and adjuvant treatment of patients with breast cancer. Eur J Cancer. 2004;40:179-81.

8. Kretschmer L, Hilgers R, Mohrle M, Balda BR, Breuninger H, Konz B, et al. Patients with lymphatic metastasis of cutaneous malignant melanoma benefit from sentinel lymphnodectomy and early excision of their nodal disease. Eur J Cancer. 2004;40:212-8.

9. Kitagawa Y, Fujii H, Mukai M, Kubota T, Otani Y, Kitajima M, et al. Radio-guided sentinel node detection for gastric cancer. Br J Surg. 2002;89:604-8.

10. Ichikura T, Morita D, Uchida T, Okura E, Majima T, Ogawa T, et al. Sentinel node concept in gastric carcinoma. World J Surg. 2002;26:318-22.
11. Hiratsuka M, Miyashiro I, Ishikawa O, Furukawa H, Motomura $\mathrm{K}$, Ohigashi $\mathrm{H}$, et al. Application of sentinel node biopsy to gastric cancer surgery. Surgery. 2001;129:335-40.

12. Rino Y, Takanashi Y, Hasuo K, Kawamoto M, Ashida A, Harada $\mathrm{H}$, et al. The validity of sentinel lymph node biopsy using dye technique alone in patients with gastric cancer. Hepatogastroenterology. 2007;54(78):1882-6.

13. Gretschel S, Bembenek A, Ulmer Ch, Hünerbein M, Markwardt J, Schneider U, et al. Prediction of gastric cancer lymph node status by sentinel lymph node biopsy and the Maruyama computer model. Eur J Surg Oncol. 2005;31(4):393-400.

14. Hohenberger P, Gretschel S. Gastric cancer. Lancet. 2003;362: 305-15.

15. La Vecchia CL, Negri E, Franceschi S, Gentile A, et al. Family history and the risk of stomach and colorectal cancer. Cancer. 1992;70:50-5.

16. Lauren P. The two histological main types of gastric carcinoma: diffuse and so-called intestinal-type carcinoma. An attempt at histo-clinical classification. Acta Pathol Microbiol Scand. 1965;64:31-9.

17. Japanese Gastric Cancer Association. Japanese classification of gastric carcinoma. 2nd English edition. Gastric Cancer 1998: 1: $10-24$.

18. Chamadol N, Wongwiwatchai J, Bhudhisawasd V, Pairojkul C. Accuracy of spiral CT in preoperative staging of gastric carcinoma: correlation with surgical and pathological findings. J Med Assoc Thai. 2008;91(3):356-63.

19. Xi WD, Zhao C, Ren GS. Endoscopic ultrasonography in preoperative staging of gastric cancer: determination of tumor invasion depth, nodal involvement and surgical resectability. World J Gastroenterol. 2003;9(2):254-7.

20. Rabin I, Chikman B, Halpern Z, Wassermann I, Lavy R, GoldDeutch R, et al. Sentinel node mapping for gastric cancer. Isr Med Assoc J. 2006;8:40-3.

21. Tajima Y, Yamazaki K, Masuda Y, Kato M, Yasuda D, Aoki T, et al. Sentinel node mapping guided by indocyanine green fluorescence imaging in gastric cancer. Ann Surg. 2009;249(1):58-62.

22. Aikou T, Higashi H, Natsugoe S, Hokita S, Baba M, Takao S, et al. Can sentinel node navigation surgery reduce the extent of lymph node dissection in gastric cancer? Ann Surg Oncol. 2001;9S:90-3.

23. Yaguchi $Y$, Ichikura $T$, Ono $\mathrm{S}$, Tsujimoto $\mathrm{H}$, Sugasawa $\mathrm{H}, \mathrm{Sa}-$ kamoto $\mathrm{N}$, et al. How should tracers be injected to detect for sentinel nodes in gastric cancer-submucosally from inside or subserosally from outside of the stomach? J Exp Clin Cancer Res. 2008;27(1):79.

24. Lee JH, Ryu KW, Kim CG, Kim SK, Choi IJ, Kim YW, et al. Comparative study of the subserosal versus submucosal dye injection method for sentinel node biopsy in gastric cancer. Eur J Surg Oncol. 2005;31:965-8.

25. Tóth D, Kathy S, Bokor L, Kincses Zs, Sebő É, Kovács I, et al. Treatment of non-palpable breast cancer and the single-agent sentinel lymph node biopsy. In: Paper presented at the 59th Congress of Hungarian Surgical Society, Debrecen, 18-20 June 2008.

26. Ishii K, Kinami S, Funaki K, Fujita H, Ninomiya I, Fushida S, et al. Detection of sentinel and non-sentinel lymph node micrometastases by complete serial sectioning and immunohistochemical analysis for gastric cancer. J Exp Clin Cancer Res. 2008;27(1):7.

27. Nakahara T, Kitagawa Y, Yakeuchi H, Fujii H, Suzuki T, Mukai $\mathrm{M}$, et al. Preoperative lymphoscintigraphy for detection of sentinel lymph node in patients with gastric cancer-initial experience. Ann Surg Oncol. 2008;15(5):1447-53.

28. Sobin LH, Wittekind C. International Union Against Cancer (UICC) TNM classification of malignant tumours. 6th ed. New York: Wiley; 2002. 